\title{
PRIME LENGTH OF CROSSED PRODUCTS
}

\author{
CHARLES C. WELSH
}

(Communicated by Donald S. Passman)

\begin{abstract}
In this paper we show that the prime length of a crossed product $R * G$, where $R$ is a right Noetherian ring and $G$ is a polycyclic-by-finite group, is bounded by the plinth length of $G$ and the prime length of $R$. We begin by considering prime ideals in group rings of finitely generated Abelian groups, and generalize a theorem of J. E. Roseblade. We then use the description of prime ideals in crossed products given by D. S. Passman to achieve the result.
\end{abstract}

\section{INTRODUCTION}

Let $R * \Gamma$ be a crossed product of the ring $R$ and the group $\Gamma$. In [3], D. S. Passman showed a correspondence between prime ideals of $R * \Gamma$ disjoint from $R$, for $R$ right Noetherian and $\Gamma$ polycyclic-by-finite, and prime ideals of a twisted group ring $F^{t} G$, where $F$ is a field related to $R$ and $G$ is a certain subgroup of $\Gamma$. He then described the primes of $F^{t} G$ using the results of J. E. Roseblade [4]. In this paper, we use Passman's description to improve the bound given in [3] (Corollary 4.3) on the prime length of such a crossed product. The main result is the extension of [4] (Theorem D) on prime ideals of group rings of Abelian groups described in the final paragraph of [3], which enables us to involve the action of $\Gamma$ on $G$.

Thorughout this paper we will assume a familiarity with the notation and results of [4], [3], and [2].

Let $\Gamma$ act on $F^{t} G$ and suppose $\Gamma$ normalizes the group of trivial units $\mathscr{G}=\left\{f \bar{g}: f \in F^{\cdot}, g \in G\right\}$. Then for $-1 \neq f \in F^{*}, \gamma \in \Gamma$, we have $(1+f)^{\gamma}=1+f^{\gamma} \in \mathscr{G}$, so $f^{\gamma} \in F^{\cdot}$, and we see that $\Gamma$ also normalizes $F^{*}$. Thus, there is an action of $\Gamma$ on $\mathscr{G} / F^{\cdot} \cong G$. We show in this situation that the $\Gamma$-prime length of $F^{t} G$ is at most the $\Gamma$-plinth length of $G$. Then, using the correspondences given in [3], we obtain a bound for the prime length of the crossed product $R * G$ in terms of the plinth length of $G$.

\section{GROUP RINGS OF ABELIAN GRoups}

We start by considering the group ring $R=F[A]$ of a finitely generated torsion-free Abelian group $A$ over a field $F$. Suppose $\Gamma$ is a group of operators

Received by the editors September 8, 1988.

1980 Mathematics Subject Classification (1985 Revision). Primary 16A27. 
on $R$. Since $A$ is an ordered group, all units of $R$ are trivial. Thus $\Gamma$ permutes $\mathscr{A}$, the group of trivial units, and we have an action of $\Gamma$ on $\mathscr{A} / F^{\circ} \cong A$.

Theorem $\mathrm{D}$ of [4] states that if $\Gamma$ normalizes $A \subset \mathscr{A}$ and centralizes $F^{\circ}$, then any faithful $\Gamma$-invariant prime ideal $P$ of $R$ is controlled by $D_{A}(\Gamma)$. Here, we allow arbitrary $\Gamma$ action, but require that no element of $A \subseteq R$ be algebraic $(\bmod P)$ over $F$, and we show that $D_{A}(\Gamma)$ controls $P$. Notice that our additional hypothesis forces $P$ to be faithful.

For the remainder of the section fix a $\Gamma$-invariant prime $P$ of $F[A]$ and let $: F[A] \rightarrow F[A] / P$ be the canonical homomorphism. If $S \subseteq T$ are integral domains, we say that $T$ is algebraic over $S$ if the field of fractions of $T$ is an algebraic extension of that of $S$. The next lemma was originally stated in the context of Roseblade's Theorem D, but the same proof works for arbitrary $\Gamma$ action.

Lemma 2.1 ([2], Lemma 5.3). Let $B \subseteq A$ be a maximal isolated orbital subgroup. If $B$ does not control $P$, then $\overline{F[A]}$ is algebraic over $\overline{F[B]}$.

Notice that the converse is also true, since if $(P \cap F[B]) F[A]=P$, then $\overline{F[A]} \cong \overline{F[B]}[A / B]$, and this is not algebraic over $\overline{F[B]}$.

If we follow the exposition of Roseblade's theorem and proof in ([2], $\S 10)$, we see that it goes through in our case, with only minor changes in the wording, until Lemma 10.3. The proof is an induction on the rank of $A$ which reduces the problem to the cases where either all elements of $A / D_{A}(\Gamma)$ are $\Gamma$-orbital or $D_{A}(\Gamma)$ is maximal isolated orbital. It is these two cases which are dealt with in Lemma 10.3. The second case, handled fairly easily there, constitutes most of the work here. We now take care of the first case.

Lemma 2.2. Let $\Gamma$ act on $F[A], P$ a $\Gamma$-invariant prime ideal. Assume that no element of $D_{A}(\Gamma)$ is algebraic $(\bmod P)$ over $F$. If, in addition, all elements of $A / D_{A}(\Gamma)$ are $\Gamma$-orbital, then $D_{A}(\Gamma)$ controls $P$.

Proof. Let $D=D_{A}(\Gamma)$. Assume $D$ does not control $P$ and consider the set of elements of $P-(P \cap F[D]) F[A]$ whose support meets the minimal number of cosets of $D$. Out of this set, pick $\alpha=\sum_{i=1}^{t} \delta_{i} a_{i}$ such that $0 \neq \delta_{i} \in F[D]$, the $a_{i}$ are in distinct cosets of $D$, and for some $j,\left|\operatorname{supp}\left(\delta_{j}\right)\right|$ is as small as possible. Without loss of generality, $j=1$. Note that $t \geq 2$. We can assume that $a_{1}=1$ and $\operatorname{tr}\left(\delta_{1}\right)=1$. Let $\Lambda \subseteq \Gamma$ centralize both $D$ and $A / D$ with $|\Gamma: \Lambda|<\infty$. Then $D_{A}(\Lambda)=D$. For $x \in \Lambda$,

$$
\alpha-\alpha^{x}=\sum_{i=1}^{t}\left(\delta_{i}-k_{i, x} \delta_{i}^{x} d_{i, x}\right) a_{i},
$$

where $k_{i, x} \in F, d_{i, x} \in D$, and $a_{i}^{x}=k_{i, x} d_{i, x} a_{i}$. Note that $k_{1, x}=d_{1, x}=1$. Either $\delta_{1}-\delta_{1}^{x}=0$, so $\operatorname{supp}\left(\alpha-\alpha^{x}\right)$ meets fewer cosets of $D$ than $\operatorname{supp}(\alpha)$, or $\delta_{1}-\delta_{1}^{x} \neq 0$. In the latter case, since $\operatorname{tr}\left(\delta_{1}\right)=1,\left|\operatorname{supp}\left(\delta_{1}-\delta_{1}^{x}\right)\right|<\left|\operatorname{supp}\left(\delta_{1}\right)\right|$. 
In both cases, the minimality of $\alpha$ yields $\alpha-\alpha^{x} \in(F[D] \cap P) F[A]$, so $\delta_{i}-k_{i, x} \delta_{i}^{x} d_{i, x} \in P$ for all $i$.

Observe that no $\delta_{i} \in F[D] \cap P$, so in the field of fractions of $\overline{F[D]}, \bar{\delta}_{i} \neq 0$ and for all $i, x$,

$$
\overline{d_{i, x}^{-1}}=\frac{\overline{k_{i, x} \delta_{i}^{x}}}{\bar{\delta}_{i}} .
$$

Now $t \neq 1$, so $a_{t} \notin D$. Thus, there is some $y \in \Lambda$ with $d_{t, y} \neq 1$. Since $\Lambda$ centralizes $D$, for all $x \in \Lambda, \overline{d_{t, x}^{-1}}$ is contained in the finite-dimensional $F$-space $V$ spanned by $1 / \delta_{t}$ times each element of $\operatorname{supp}\left(\delta_{t}\right)$. Also, $\left(\overline{d_{t, y}}\right)^{-n} \in F\left(\overline{d_{t, y^{n}}^{-1}}\right) \subseteq V$ for all integer exponents $n$, so the powers of $\overline{d_{t, y}^{-1}}$ are not linearly independent over $F$. Hence $\overline{d_{t, y}^{-1}}$ is algebraic over $F$, contradicting $d_{t, y} \neq 1$.

For the case where $D_{A}(\Gamma)$ is maximal isolated obital, we will assume that $D_{A}(\Gamma)$ does not control $P$ and show that each element of $\Gamma$ must act finitely on $A$. We will then apply the following lemma from the theory of linear groups (see [5], §9.1).

Lemma 2.3. Let $G$ be a periodic subgroup of $\operatorname{GL}(n, Q)$. Then $G$ is finite.

Lemma 2.4. Let $\Gamma$ be a cyclic group of operators on $F[A], P$ a $\Gamma$-invariant prime ideal. Suppose $D_{A}(\Gamma)$ is maximal isolated orbital and does not control $P$. Then some nonidentity element of $[A, \Gamma]$ is algebraic $(\bmod P)$ over $F$.

Proof. Let $D=D_{A}(\Gamma)$. By replacing $\Gamma$ with a subgroup of finite index, we may assume that $\Gamma$ centralizes $D$. Let $\Gamma=\langle g\rangle$. Then $[A, \Gamma]=[A, g]=\{(a, g)$ : $a \in A\}$.

Suppose $[A, \Gamma] \cap D>1$. Say $1 \neq b=[a, g] \in D$. By Lemma $2.1, \overline{F[A]}$ is algebraic over $\overline{F[D]}$. Suppose $\bar{b}$ is not algebraic over $F$. Extend $\bar{b}$ to a transcendence basis $\left\{\bar{b}=\overline{d_{0}}, \overline{d_{1}}, \ldots, \overline{d_{n}}\right\}$ for $\overline{F[D]}$, and thus for $\overline{F[A]}$, over $F$, with each $d_{i} \in D$. Then $\overline{F[A]}$ is algebraic over $\overline{F\left[d_{0}, d_{1}, \ldots, d_{n}\right]}$, and, $\bmod P, a$ satisfies an algebraic equation over $F\left[d_{0}, d_{1}, \ldots, d_{n}\right]$. Let $\sum_{i=0}^{t} \delta_{i} a^{i} \in P$ be a relation of minimal degree. Note that $\delta_{0} \neq 0$ and $t \geq 1$. Another relation is $\sum_{i=0}^{t} \delta_{i}^{g}\left(a^{i}\right)^{g} \in P$. In $F[A],\left(a^{i}\right)^{g}=f^{i} b^{i} a^{i}$ for some $f \in F$, so $\sum_{i=0}^{t} f^{i} \delta_{t} \delta_{i}^{g} b^{i} a^{i} \in P$ and $\sum_{i=0}^{t} f^{t} \delta_{t}^{g} b^{t} \delta_{i} a^{i} \in P$ are two minimal relations with the same coefficient of $a^{t}$. Thus, if we look at the term with $i=0$, we see that $\delta_{t} \delta_{0}^{g}=f^{t} \delta_{t}^{g} \delta_{0} b^{t}$. Notice that since $\Gamma$ centralizes $D$, $\Gamma$ normalizes $F\left[d_{0}, d_{1}, \ldots, d_{n}\right]$ and stablizes any $F$-subspace spanned by a monomial. Thus the degree in $d_{0}$ of $\delta_{i}$ is the same as that of $\delta_{i}^{g}$. But $t \geq 1$ and $d_{0}=b$, so the degree in $d_{0}$ of $\delta_{t} \delta_{0}^{g}$ is less than the degree in $d_{0}$ of $f^{t} \delta_{t}^{g} \delta_{0} b^{t}$, a contradiction. It follows that $\bar{b}$ must be algebraic over $F$.

Now assume that $[A, \Gamma] \cap D=1$. We consider the cases $F[[A, \Gamma]] \cap P=0$ and $F[[A, \Gamma]] \cap P \neq 0$. 
Suppose $F[[A, \Gamma]] \cap P=0$. Note that since $[A, \Gamma]$ is torsion-free, $F[[A, \Gamma]]$ is a localization of a polynomial ring over $F$, and is therefore a unique factorization domain. Again, since $\overline{F[A]}$ is algebraic over $\overline{F[D]}$, we can pick a transcendence basis $\left\{\bar{d}_{0}, \overline{d_{1}}, \ldots, \overline{d_{n}}\right\}$ for $\overline{F[A]}$ over $F$ with $d_{i} \in D$. Let $E=\left\langle d_{0}, d_{1}, \ldots, d_{n}\right\rangle \subseteq D$ and set $B=[A, \Gamma] \times E . \overline{F[B]}$ is algebraic over $\overline{F[E]}$, so for any $1 \neq a \in[A, \Gamma]$ there is a relation $\sum \beta_{i} a^{i} \in F[B] \cap P$, $\beta_{i} \in F[E]$. Note that $\sum \beta_{i} a^{i} \neq 0$, since $a$ is independent over $F[E]$. Thus $F[B] \cap P \neq 0$. Pick $0 \neq \alpha \in F[B] \cap P$ with $\operatorname{supp}(\alpha)$ meeting the minimal number of cosets of $[A, \Gamma]$, say $\alpha=\sum_{i=1}^{t} \alpha_{i} e_{i}, 0 \neq \alpha_{i} \in F[[A, \Gamma]], e_{i} \in E$. Note that $t>1$, since neither $\alpha_{1}$ nor $e_{1}$ is in $P$.

Suppose that the $\alpha_{i}$ have a common prime divisor $\tau \in F[[A, \Gamma]]$. Then $\alpha=\tau \sum_{i=1}^{t} \alpha_{i}^{\prime} e_{i}$. Since $F[[A, \Gamma]] \cap P=0, \tau \notin P$ so $0 \neq \alpha^{\prime}=\sum_{i=1}^{t} \alpha_{i} e_{i} \in$ $P \cap F[B]$, and we can replace $\alpha$ with $\alpha^{\prime}$. Thus we can assume that the $\alpha_{i}$ have no common prime divisor.

Note that $\Gamma$ normalizes $[A, \Gamma]$. Let $x \in \Gamma$. Since $\Gamma$ centralizes $E \subseteq D$, in $F[B], e_{i}^{x}=f_{i} e_{i}$ for some $f_{i} \in F$. For each $j, \sum_{i=1}^{t} f_{j} \alpha_{j}^{x}\left(\alpha_{i} e_{i}\right)$ and $\sum_{i=1}^{t} \alpha_{j}\left(\alpha_{i}^{x} f_{i} e_{i}\right)$ are two minimal elements of $P \cap F[B]$ with the same coefficient of $e_{j}$. Thus, for all $i, j, x$,

$$
f_{j} \alpha_{j}^{x} \alpha_{i}=f_{i} \alpha_{j} \alpha_{i}^{x}
$$

Fix $i$ and let $\pi$ be a prime divisor of $\alpha_{i}$ with $\operatorname{tr}(\pi)=1$. Since $t>1$, there is a $j$ such that $\pi$ does not divide $\alpha_{j}$. Thus, from $(*)$, we see that $\pi \mid \alpha_{i}^{x}$, so $\pi^{x^{-1}} \mid \alpha_{i}$, and $\Gamma$ permutes the finite number of associativity classes of prime divisors of $\alpha_{i}$. Now, all units of $F[[A, \Gamma]]$ are trivial, so for any prime $\tau$ of $F[[A, \Gamma]]$, the associativity class of $\tau$ is $\left\{f a \tau: f \in F^{\circ}, a \in[A, \Gamma]\right\}$, and it is clear that the number of trace one primes associated to $\tau$ is $|\sup (\tau)|$. For any $x \in \Gamma, \operatorname{tr}\left(\pi^{x}\right)=1$, so it follows that the $\Gamma$-orbit of $\pi$ is fintie. We now have that $\Gamma$ permutes the finite set $\bigcup_{x \in \Gamma} \operatorname{supp}\left(\pi^{x}\right)$, so $\pi \in F[D]$, a contradiction. It must be that $\alpha_{i}$ has no prime divisors, so $\alpha_{i}=l_{i} a_{i}$ for some $l_{i} \in F$, $a_{i} \in[A, \Gamma]$.

Without loss of generality, $f_{1}=\alpha_{1}=1$, so with $j=1,(*)$ becomes

$$
l_{i} a_{i}=f_{i} l_{i}^{x} a_{i}^{x} .
$$

It now becomes evident that in the action of $\Gamma$ on $A, \Gamma$ fixes each $a_{i}$, so $a_{i} \in D \cap[A, \Gamma]=1$. Thus, $\alpha=\sum_{i=1}^{t} l_{i} e_{i} \in P$, contradicting the algebraic independence of the $\overline{d_{i}}$.

Finally, if $D \cap[A, \Gamma]=1$ and $F[[A, \Gamma]] \cap P \neq 0$, we apply Lemma 2.1. Since $F[A]$ is commutative, $P \cap F[[A, \Gamma]]$ is prime. Since $D[A, \Gamma]$ is $\Gamma$-orbital and strictly contains $D, D[A, \Gamma]$ has finite index in $A$, so $D$ is maximal isolated orbital in $D[A, \Gamma] . \quad D[A, \Gamma] / D$ is $\Gamma$-isomorphic to $[A, \Gamma]$, so $D_{[A, \Gamma]}(\Gamma)=$ $D \cap[A, \Gamma]=1$ is maximal isolated orbital in $[A, \Gamma] .1$ certainly does not control $P \cap[A, \Gamma]$, so by Lemma $2.1, \overline{[A, \Gamma]}$ is algebraic over $F$. 
Lemma 2.5. Let $\Gamma$ be a group of operators on $F[A], P$ a $\Gamma$-invariant prime ideal. Suppose that $D_{A}(\Gamma)$ is maximal isolated orbital and that no nonidentity element of $A$ is algebraic $(\bmod P)$ over $F$. Then $D_{A}(\Gamma)$ controls $P$.

Proof. Let $D=D_{A}(\Gamma)$. Assume that $\Gamma$ centralizes $D$ and that $D$ does not control $P$. Let $g \in \Gamma, X=D_{A}\langle g\rangle$. We will show that $A=X$. Note that $D \subseteq X$.

Suppose $A \neq X . A / X$ is torsion-free, so there is a $\langle g\rangle$-orbital $B, X<B \subseteq$ $A$, and $X$ maximal isolated $\langle g\rangle$-orbital in $B$. Let $1 \neq H \subseteq\langle g\rangle$ normalize $B$. Since $|\langle g\rangle: H|<\infty, X=D_{B}(H)$. Since $D$ does not control $P, \overline{F[A]}$ is algebraic over $\overline{F[D]}$, so $\overline{F[B]}$ is algebraic over $\overline{F[X]}$. Thus $X$ cannot control $P \cap F[B]$, and, by Lemma 2.4 , some nonidentity element of $[B, H]$ is algebraic $(\bmod P)$ over $F$, a contradiction. We have $A=X$, so some finite index subgroup of $\langle g\rangle$ centralizes $A$, and $\langle g\rangle$ acts finitely on $A$.

Now consider the rational vector space $V=A \otimes_{Z} Q$. The action of $\Gamma$ on $A$ can be extended to an action of $\Gamma$ on $V$, so we have a map $\phi: \Gamma \rightarrow \operatorname{End}_{F}(V)$. $V$ is finite dimensional, and we have shown that $\phi(\Gamma)$ is periodic. Thus, by Lemma 2.3, $\phi(\Gamma)$ is finite. It follows that $\Gamma$ has a subgroup of finite index that centralizes $A$, so $D=A$, contradicting $D<A$.

We can now replace ([2], Lemma 10.3) with Lemmas 2.2 and 2.5 to get

Theorem 2.6. Let $A$ be a finitely generated torsion-free Abelian group, $F$ a field, and $\Gamma$ a group of operators of $F[A]$. If $P$ is a $\Gamma$-invariant prime ideal of $F[A]$ such that no nonidentity element of $A$ is algebraic $(\bmod P)$ over $F$, then $D_{A}(\Gamma)$ controls $P$.

\section{T-PRIME LENGTHS}

Recall that $F^{t} G$ is a twisted group algebra of $G$ over $F$. We now obtain a bound on the $\Gamma$-prime length of $F^{t} G$. For $R$ a ring and $G$ a group, denote by $\operatorname{pr}_{\Gamma}(R)$, the $\Gamma$-prime length of $R$, and by $\mathrm{p}_{\Gamma}(G)$, the $\Gamma$-plinth length of $G$.

Theorem 3.1. Let $G$ be a polycyclic-by-finite group, and $\Gamma$ a group of operators on $F^{t} G$ which normalizes the group of trivial units $\mathscr{G}$. Then $\operatorname{pr}_{\Gamma}\left(F^{t} G\right) \leq \mathrm{p}_{\Gamma}(G)$.

Proof. Since every ideal of $F^{t} G$ is $\mathscr{G}$ invariant, we may assume that $\mathscr{G}$ is a normal subgroup of $\Gamma$. Then anything which is $\Gamma$ invariant is also $G$ invariant. For $X$ a subgroup of $\mathscr{G}$, denote by $\bar{X}$ the image of $X \bmod F^{*}$. The arguments of [4] (\$8.1) also work for twisted group rings. These, combined with [3] (Lemma 4.1), allow us to replace $G$ and $\Gamma$ by subgroups of finite index. In particular, by [3] (Proposition 3.2), we can replace $G$ by the subgroup nio $^{2}(G)$. We will associate to each $\Gamma$-orbital prime $P$ of $F^{t} G$ a non-negative integer $\tau(P)$. For this we require some notation.

First, we define $P^{t}=\{f \bar{g} \in \mathscr{G}: 1-f \bar{g} \in P, f \in F, g \in G\}$. Note that $P^{t} \triangleleft \mathscr{G}$, so $\overline{P^{t}} \triangleleft G$. Define $\nabla_{G}^{t}\left(P^{t}\right)$ as follows. If $X / P^{t}=\Delta\left(\mathscr{G} / P^{t}\right)$, then $\nabla_{G}^{t}\left(P^{t}\right)=\bar{X}$. 
Now, let $N=\nabla_{G}^{t}\left(P^{t}\right)$. By [3] (Propositions 3.6 and 4.2), we know that $P=L^{G}$ for some prime $L$ of $F^{t} N$ unique up to conjugation by $\mathscr{G}$. From the discussion following [3] (Proposition 3.6), we see that there is an $A \subseteq N$ such that $A / \overline{P^{t}}$ is torsion-free Abelian, $|N: A|<\infty$, and $F^{t}\left(A / \overline{P^{t}}\right)$ is a central subring of $F^{t}\left(N / \overline{P^{t}}\right)$. Since $N \triangleleft G$, we can choose $A \triangleleft G$.

Let $Q$ be the image of $L$ in $F^{t}\left(N / \overline{P^{t}}\right)$. Then $Q \cap F^{t}\left(A / \overline{P^{t}}\right)$ is prime, so we can consider the set $B / \overline{P^{t}}$ of elements of $A / \overline{P^{t}}$ which are algebraic $\left(\bmod Q \cap F^{t}\left(A / \overline{P^{t}}\right)\right)$ over $F$. Clearly $B$ is a subgroup of $A$, and $A / B$ is torsion-free.

Since $P$ is $\Gamma$-orbital, $L$ is, so there is a subgroup $\Gamma_{0}$ of finite index in $\Gamma$ which stabilizes $P, L$, and anything uniquely determined by $P$ or $L$ including $N$. Since $A$ has finite index in $N$, there is a subgroup $\Lambda$ of finite index in $\Gamma_{0}$ which stabilizes $A$, and hence $\Lambda$ also stabilizes $B$.

Now, let $D / B=D_{A / B}(\Lambda)$. Since $P^{t}$ maps to $F, \bmod L \cap F^{t} D$, $F^{t} D /\left(L \cap F^{t} D\right)$ is a commutative integral domain, so it makes sense to look at its transcendence degree over $F$. Define $\tau(P)$ as follows:

$$
\tau(P)=\text { t.d. }_{F} \frac{F^{t} D}{L \cap F^{t} D}+\mathrm{p}_{\Lambda}(G / D) .
$$

Notice that since the image of $B$ in $F^{t} D /\left(L \cap F^{t} D\right)$ is algebraic over $F$, t.d. $F^{t} D /\left(L \cap F^{t} D\right) \leq h(D / A)$, where $h$ is the Hirsch number. But $\Lambda$ acts finitely on $D / B$, so $h(D / B)=p_{\Lambda}(D / B)$ and $\tau(P) \leq \mathrm{p}_{\Lambda}(D / B)+\mathrm{p}_{\Lambda}(G / D) \leq$ $\mathrm{p}_{\Lambda}(G)=\mathrm{p}_{\Gamma}(G)$.

Suppose that $A_{1}$ is another choice for $A$ in the definition of $\tau$. To show that both give the same value of $\tau$, it suffices to show that, say, using $A$ gives the same result as using $A_{2}=A \cap A_{1}$. We may replace $\Lambda$ by $\Lambda_{2} \subseteq \Lambda$ of finite index which stabilizes $A_{2}$. Let $B_{2}$ and $D_{2}$ be the subgroups of $A_{2}$ which correspond to $B$ and $D$.

Since $B_{2}=B \cap A_{2},\left|B: B_{2}\right|<\infty$. Thus, $D / B_{2}=D_{A / B_{2}}\left(\Lambda_{2}\right) . D_{2} / B_{2}=$ $\left(D / B_{2}\right) \cap\left(A_{2} / B_{2}\right)$, so $\left|D: D_{2}\right|<\infty$. Now, if $T$ is a transversal for $D_{2}$ in $D$, then $F^{t} D$ is generated over $F^{t} D_{2}$ by $T$, and some power of each element of $D$ is in $D_{2}$. Thus, $F^{t} D /\left(L \cap F^{t} D\right)$ is algebraic over $F^{t} D_{2} /\left(L \cap F^{t} D_{2}\right)$. Clearly $\mathrm{p}_{\Lambda}(G / D)=\mathrm{p}_{\Lambda_{2}}\left(G / D_{2}\right)$, so it follows that the value of $\tau$ is independent of the choice of $A$.

Suppose $P<\widehat{P}$ are two $\Gamma$-orbital primes of $F^{t} G$. Our goal is to show that $\tau(\widehat{P})<\tau(P)$. We can assume that $\Gamma$ stabilizes $P, \widehat{P}$, and all of the corresponding structures for each as described above. Let $I=\left(x-1: x \in P^{t}\right)$. Then $I \subseteq P<\widehat{P}$ and $F^{t} G / I \cong F^{t}\left(G / \overline{P^{t}}\right)$, so we can assume that $P^{t}=1$. Thus $A$ is torsion-free Abelian and $F^{t} A$ is commutative, so $F^{t} A \cong F[A]$.

Recall that $F^{t} B$ is central in $F^{t} D$. Let $J=\left(P \cap F^{t} B\right) F^{t} D$. Then $F^{t} D / J \cong$ $F_{0}^{t}(D / B)$, where $F_{0}$ is the field $F[B] /(P \cap F[B])$, an algebraic extension of $F$. Working in this new crossed product allows us to assume that $B=1$. 
Let $\widehat{N}, \widehat{L}, \widehat{\Lambda}, \widehat{A}, \widehat{B}$, and $\widehat{D}$ be the structures associated with $\widehat{P}$. Since $N \subseteq$ $\widehat{N}$, we can replace $A$ by its finite index subgroup $A \cap \widehat{A}$. Thus we can assume that $A \subseteq \widehat{A}$, so $D \subseteq \widehat{D}$. We may also assume tht $\widehat{\Lambda}=\Lambda$. Since $L$ and $\widehat{L}$ are $\Gamma$-invariant, $L^{\widehat{N}}=L\left(F^{t} \widehat{N}\right) \subseteq \widehat{P} \cap F^{t} \widehat{N}$. Suppose $M$ is a minimal covering prime of $\widehat{P} \cap F^{t} \widehat{N}$. Then $M^{G}=\widehat{P}$, so $M$ is $\mathscr{G}$-conjugate to $\widehat{L}$. But then $M=\widehat{L}$, and it follows that $L \subseteq \widehat{L}$.

Now, $F^{t} \widehat{D} /\left(\widehat{L} \cap F^{t} \widehat{D}\right)$ is algebraic over a subring generated by the image of $F^{t}(D \widehat{B})$ and $h(\widehat{D} / D \widehat{B})$ other generators. Moreover, $\Lambda$ acts finitely on $\widehat{D} / D \widehat{B}$, so $h(\widehat{D} / D \widehat{B})=\mathrm{p}_{\Lambda}(\widehat{D} / D \widehat{B})$. $\widehat{B}$ is algebraic $\left(\bmod \widehat{L} \cap F^{t} D \widehat{B}\right)$ over $F$, so

$$
\text { t.d. } \frac{F^{t}(D \widehat{B})}{\widehat{L} \cap F^{t}(D \widehat{B})}=\text { t.d. } \frac{F^{t} D}{\widehat{L} \cap F^{t} D}
$$

and

Thus,

$$
\text { t.d. } F_{F} \frac{\widehat{D}}{\widehat{L} \cap F^{t} \widehat{D}} \leq \text { t.d. }_{F} \frac{F^{t} D}{\widehat{L} \cap F^{t} D}+\mathrm{p}_{\Lambda}(\widehat{D} / D \widehat{B}) \text {. }
$$

$$
\begin{aligned}
\tau(\widehat{P}) & =\operatorname{t.d} ._{F} \frac{F^{t} \widehat{D}}{\widehat{L} \cap F^{t} \widehat{D}}+\mathrm{p}_{\Lambda}(G / \widehat{D}) \leq \operatorname{t.d}_{F} \frac{F^{t} D}{\widehat{L} \cap F^{t} D}+\mathrm{p}_{\Lambda}(G / D \widehat{B}) \\
& \leq \operatorname{t.d}_{F} \frac{F^{t} D}{L \cap F^{t} D}+\mathrm{p}_{\Lambda}(G / D)=\tau(P) .
\end{aligned}
$$

Assume that the second inequality of $(* *)$ is actually an equality. Then both

$$
\text { t.d. } \frac{F^{t} D}{\widehat{L} \cap F^{t} D}=\text { t.d. } F \frac{F^{t} D}{L \cap F^{t} D}
$$

and

$$
\mathrm{p}_{\Lambda}(G / D \widehat{B})=\mathrm{p}_{\Lambda}(G / D) .
$$

From (1), since $F^{t} D$ is commutative, $\widehat{L} \cap F^{t} D=L \cap F^{t} D$. From (2), $D \widehat{B} / D$ is finite. Mod $\widehat{L} \cap F^{t} D=L \cap F^{t} D, \widehat{B} \cap D$ is algebraic over $F$, so $D \cap \widehat{B} \subseteq B=1$. Hence $\widehat{B}$ is finite. But then $\widehat{\widehat{P}}^{t} \subseteq \widehat{B}$ is finite, so $\widehat{N}=\nabla_{G}^{t}\left(\widehat{P}^{t}\right)=\Delta^{t}(G)$, and we can assume that $A=\widehat{A}$. Thus, $\widehat{B} \subseteq D$, so $\widehat{B}=1$.

Now, since $F^{t} D \cong F[D]$, it follows from Theorem 2.6 that $L \cap F^{t} D$ and $\widehat{L} \cap F^{t} D$ are controlled by $D=\widehat{D}$. Hence

$$
L \cap F^{t} A=\left(L \cap F^{t} D\right) F^{t} A=\left(\widehat{L} \cap F^{t} D\right) F^{t} A=\widehat{L} \cap F^{t} A,
$$

and since $A$ has finite index in $N, L=\widehat{L}$. Thus, by [3] (Theorem 3.6), $P=\widehat{P}$ is a contradiction. It must be that the second inequality in $(* *)$ is strict, so $\tau(\widehat{P})<\tau(P)$.

Now, if

$$
P_{0}<P_{1}<\cdots<P_{n}
$$

is a chain of $\Gamma$-prime ideals, then

$$
0 \leq \tau\left(P_{0}\right)<\tau\left(P_{1}\right)<\cdots<\tau\left(P_{n}\right) \leq \mathrm{p}_{\Lambda}(G),
$$


so $n \leq \mathrm{p} \Lambda(G)$, and the theorem is proved.

Let $G$ be polycyclic-by-finite, $R$ a prime right Noetherian ring. In [3], Passman shows that there is a field $F$ related to $R$ and a normal subgroup $G_{\text {inn }}$ of $G$ such that the primes of $R * G$ correspond to the $G$-primes of $F^{t} G_{\text {inn }}$. This correspondence is used to obtain a bound ([3], Corollary 4.3) on the prime length of $R * G$. The bound is in terms of the prime length of $R$ and the Hirsch number of $G$. One step in the proof is to bound $\operatorname{pr}_{\Gamma}\left(F^{t} G_{\text {inn }}\right)$ by $h\left(G_{\text {inn }}\right) \leq h(G)$. In light of Theorem 3.1 , we can replace $h(G)$ and $h\left(G_{\text {inn }}\right)$ by $\mathrm{p}_{G}(G)$ and $\mathrm{p}_{G}\left(G_{\text {inn }}\right)$ to get

Corollary 3.2. Let $R$ be right Noetherian and $G$ polycyclic-by-finite. Then

$$
\operatorname{pr}(R * G)<\left(\mathrm{p}_{G}(G)+1\right)(\operatorname{pr}(R)+1) .
$$

\section{ACKNOWLEDGMENT}

The author would like to thank Professor Passman for his invaluable direction and suggestions.

\section{REFERENCES}

1. D. S. Passman, The algebraic structure of group rings, Wiley-Interscience, New York, 1977.

2. _ Group rings of polycyclic groups, in Group Theory: Essays for Phillip Hall (K. H. Gruenberg and J. E. Roseblade editors), Academic Press, New York, 1984.

3. __ Prime ideals in polycyclic crossed products, Trans. Amer. Math. Soc. (2) 301 (1987), 737-759.

4. J. E. Roseblade, Prime ideals in group rings of polycyclic groups, Proc. London Math. Soc. (3) 36 (1978), 385-447.

5. B. A. F. Wehrfritz, Infinite linear groups, Springer-Verlag, New York, 1973.

UNIVERSity OF Wisconsin-MAdison, Madison, Wisconsin 53706 